\title{
„Sie gestalten! Und wenn Sie nicht gestalten, gestaltet es Sie!“
}

Am 27. und 28. Januar 2017 fand im Magnus-Haus Berlin bereits zum dritten Mal der Basiskurs zur Veranstaltungsreihe „Führungskompetenzen für Radiologen“ der Röntgen Akademie statt. Das Kursprogramm war breit gefächert und stellte eine ausgewogene Mischung aus Vortrag und praktischen Übungseinheiten dar. Die Themen reichten dabei von aktuellen Ansätzen der Führungsforschung über Führungsstile, -logiken und -rollen, Mitarbeiter- und Motivationsgespräche bis zu Bewerbungs- und Auswahlverfahren für Führungspositionen.

Sechzehn Radiologinnen und Radiologen aus allen Teilen der Republik und der benachbarten Schweiz hatten den Weg nach Berlin gefunden, um sich durch ein zweitägiges Programm führen zu lassen und selbst Führungsinstrumente zu erproben. Mit im Gepäck: praktische Erfahrungen, Kompetenzen, Probleme und Anforderungen aus nahezu allen Bereichen radiologi- schen Arbeitens sowie eine klare Erwartungshaltung: „Hoffentlich ist das ein Kurs, in dem man auch etwas lernen kann.“

Vertreter von Universitätskliniken, Medizinischen Versorgungszentren, Kliniken in kommunaler Trägerschaft und von Gemeinschaftspraxen saßen Seite an Seite, um sich intensiv mit dem Thema Führung in seinen theoretischen und praktischen Dimensionen auseinanderzusetzen. Seminarleiter Jens Hollmann, langjähriger Coach und Berater für Ärzte und Klinikleitungen sowie Gründer und Inhaber von medplus-kompetenz, stellte gleich zu Beginn klar, dass ein Seminar wie dieses vor 20 Jahren sicherlich nicht notwendig gewesen wäre. Dies habe vor allen Dingen mit den sich stark veränderten Erwartungen zu tun, denen Führungskräfte heute ausgesetzt seien. Von Klinikleitungen werde nicht länger der reine Medizinexperte, sondern immer mehr auch eine Management-Persönlichkeit gesucht, die unternehmerisch denken und handeln könne. Dem stehe auf der anderen Seite eine Mitarbeitergeneration gegenüber, die nicht nur Hierarchien zunehmend kritisch sähe, sondern auch den Verzicht auf materielle Werte mit einem Mehr an privater Zeit, Gestaltungsspielräumen und der Teilhabe an Wissen verbinden würde.

\section{„Führung, die nicht führt, hinterlässt nie ein Vakuum"}

Doch wie lässt sich gute Führung heute umsetzen? Die Antwort darauf hat zunächst einmal auch etwas mit dem Stil zu tun, der zu der eigenen Persönlichkeit passt, insbesondere aber von den Zielen abhängt, die erreicht werden sollen. Autoritär, affiliativ, visionär oder demokratisch? Es gibt viele Möglichkeiten, wie man führen kann, wobei manche Führungsstile in bestimmten Arbeitskontexten eher zum gewünschten $\mathrm{Er}$ gebnis führen als andere. Was hingegen alle Stile verbindet bzw. einen wesentlichen $\mathrm{Er}$ - 


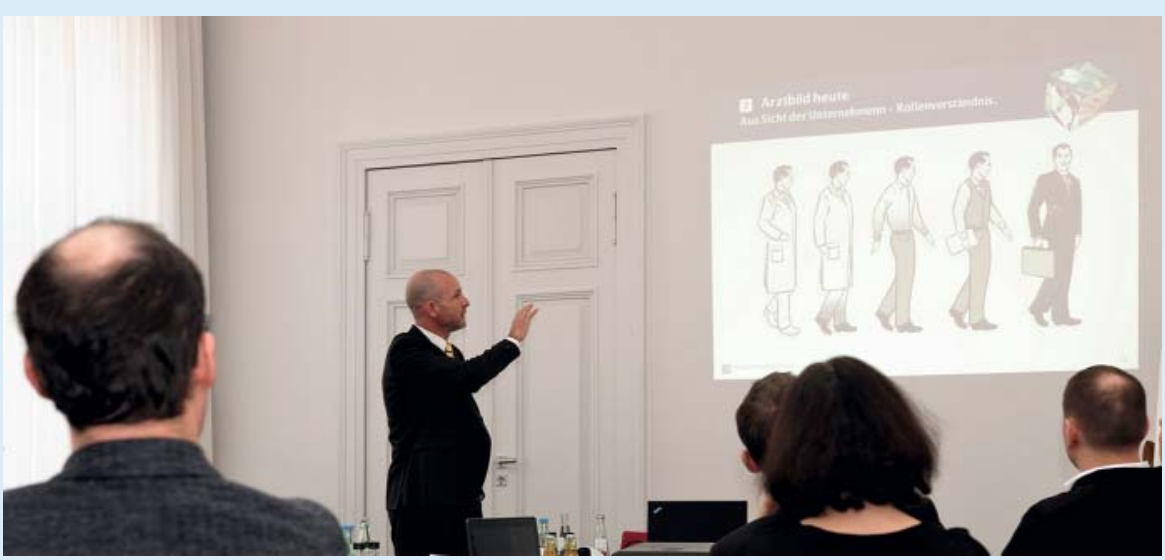

Johannes Zolk von Odgers Berndtson skizziert den evolutionären Transformationsprozess, den ärztliche Führungskräfte aus Sicht von Unternehmen durchlaufen.

folgsfaktor beschreibt, ist der passende Mix aus strukturalen und personalen Führungselementen. Abhängig von der jeweiligen Problemlage sollte das Vorgehen gezielt struktural oder personal angelegt sein. Es gilt aber auch: Je mehr Mitarbeiter geführt werden, desto wichtiger sind strukturale Elemente als entlastende Faktoren, um Führung auch wirklich über einen längeren Zeitraum hinweg bewältigen und gestalten zu können. Als aktuelles Beispiel aus dem Arbeitsalltag diente den Seminarteilnehmern der Befundbericht. Was tun, wenn ein Mitarbeiter durch formale Mängel bei dessen Erstellung wiederholt negativ auffällt? Zu den diskutierten strukturellen Lösungsansätzen zählten beispielsweise die Einrichtung eines Schreibbüros, Musterblätter oder auch die Bedarfserhebung auf Zuweiserseite, um gegebenenfalls Befunde knapper und standardisierter anlegen zu können.

Die Frage nach dem passenden Führungsstil ist wesentlich von der eigenen Führungspersönlichkeit abhängig. Versteht man sich beispielsweise als Stratege, eignet man sich in besonderer Weise als Mentor für einen Wandel des Unternehmens bzw. der Abteilung. Der Diplomat wiederum funktioniert besonders als ein verbindendes Glied, um ein Gemeinschaftsgefühl aufzubauen und zu verstetigen. Doch auch die Mitarbeiter in einer Abteilung verfügen über ganz unterschiedliche Persönlichkeitsprofile. Hier kommt es laut Hollmann auf die richtige Mischung an. So könnten z. B. Strategen und Experten zusammen mit einem oder mehreren Individualisten als „Musterbrecher“ den Kern einer sehr gut funktionierenden Abteilungsstruktur ausmachen.

Der Blick der Seminarteilnehmer auf die eigene erlebte Arbeitswirklichkeit zeigt, dass hier alles möglich ist: Macher mit einem autoritären Führungsstil existieren ebenso wie auch demokratische, partizipative Modelle. Der Blick auf die eigene Persönlichkeit wiederum führte zu der Erkenntnis, dass man auch mehrere Persönlichkeitsmerkmale auf sich vereinen konnte.

Über allem steht jedoch für Hollmann der Grundsatz, dass immer jemand führt. Leere Räume, die sich aus der eigenen Zurückhaltung oder Passivität ergäben, würden von anderen genutzt. Dies könne ebenfalls zum Wohle einer Abteilung sein oder aber nur dem persönlichen Profilierungsstreben dienen.

\section{Zentrales Führungsinstru- ment: das Mitarbeiter- gespräch}

Mitarbeitergespräche sind nicht überall fester Bestandteil von Führung. Das ergab eine Abfrage bei den Kursteilnehmern. Nur weniger als die Hälfte konnte auf konkrete Erfahrungswerte zurückgreifen. Mangelnde oder unzureichende Vorbereitung, fehlende Zielausrichtung oder aber schlicht die falsche personelle Besetzung - es zeigte sich zudem sehr schnell, dass auch die erlebte Praxis oftmals nicht frei von Fehlern und Unzulänglichkeiten ist. Wie ein Mitarbeitergespräch idealtypisch verlaufen kann und welcher vor- bereitenden Schritte es hierzu bedarf, stellte Jens Hollmann in einem Kurzreferat vor, um dann direkt in den praktischen Teil überzugehen. In simulierten Mitarbeitergesprächen konnten die Anwesenden in die Rolle des Vorgesetzten wie auch des Mitarbeiters schlüpfen und sich in Gesprächs- und Fragetechniken erproben.

\section{„Welche Vision würden Sie verfolgen?"}

Bewerbungs- und Auswahlverfahren für Chefarztpositionen standen im Mittelpunkt eines Vortrags von Johannes Zolk von Odgers Berndtson, einem der führenden Anbieter von Executive-Search-Dienstleistungen. Dabei verwies er insbesondere auf das stark veränderte Arztbild von Unternehmen, das zunehmend von betriebswirtschaftlichen Anforderungen geprägt sei, und erläuterte am konkreten Beispiel den idealtypischen Ablauf eines Bewerbungsund Auswahlverfahrens. Entlang verschiedener Fragestellungen wie „Welche ökonomischen Auswirkungen ergeben sich durch das medizinische Leistungsspektrum unter Ihrer Führung?" oder "Wie wollen Sie sich von den Wettbewerbern differenzieren?“ hatten die anwesenden Radiologen anschließend Gelegenheit, sich vor dem Auditorium zu präsentieren und der konstruktiven Kritik von Kolleginnen und Kollegen zu stellen.

\section{Nach dem Basiskurs ist vor der Modulveranstaltung}

Die Teilnehmerinnen und Teilnehmer zeigten sich insgesamt sehr zufrieden mit dem, was sie in den letzten zwei Tagen gelernt und erlebt hatten. Einige kündigten bereits beim Verlassen des Seminarraums ihr Erscheinen bei einem der nachfolgenden Modulveranstaltungen an. Gelegenheit hierzu bietet sich am 23./24. Juni 2017 (Modul 2 - Konfliktmanagement) und am 20./21. Oktober 2017 (Modul 3 - Changemanagement). Die Teilnahme an einem Basiskurs ist Voraussetzung, um eine Modulveranstaltung belegen zu können. Der nächste Basiskurs ist für den 15./16. September 2017 geplant.

Bis dahin bleibt es dabei: „Sie haben die Mitarbeiter, die Sie verdient haben!“ 